\title{
A Simulation Study for Computing the Emissivity of Clouds
}

\author{
S. Seker, M. E. Aydemir, G. Apaydin
}

In this study, propagation through various distributions of lossy particles in clouds is investigated. Clouds may contain several types of crystal forms which can be modelled in the physical optics scattering region such as thin long cylinders and flat plate. The bistatic scattering patterns and emissivities of various types of clouds are computed for waves of selected polarizations passing through clouds with specified sizes, shapes and distributions. The results are in good agreement with the literature.

Keywords: scattering, emissivity, physical optics, forward scattering theorem.

\section{INTRODUCTION}

The propagation effects due to atmospherical interference in higher microwave bands have started to be investigated during recent years. It was seen that rain and the formation of ice crystals in clouds are the dominant physical characteristics affecting channel characteristics [1]. In parallel, modeling efforts have been intensified to simulate scattering and emission from different types of clouds [2]. In the literature there are few studies that focus on the scattering properties of ice crystals at millimeter-wave frequencies. Models of microwave emission developed in the past decade are based on a single scattering approach. Scatterers are assumed to be equal in dimensions, permittivity and shape, so that the cloud is characterized by single scattering albedo and the optical thickness $\tau$.

Recent works indicate that the present trend is to describe cloud mechanisms in more realistic detail. In this study, a cloud is modelled as a random collection of discrete scatterers. Flat plate crystals are modelled by circular discs and long cylinder bullets are modelled by circular cylinders. Formulas to compute the absorption cross-section of random lossy dielectric dics and cylinders in the physical optics approximation are derived, and their validity is checked by means of the forward scattering theorem. The bistatic scattering patterns and emissivity plots of various clouds are drawn using the statistical cloud parameters from some published papers.

\section{Definitions and approximations}

All substances emit radiation with an intensity proportional to the temperature according to the Stefhan-Boltzmann law. However, this law represents the upper limit in radiation intensity that a substance could emit for a particular temperature. Such a substance is normally called a 'black body'. In order to compare the actual to the theoretical emission, the emissivity concept is defined as the ratio of the actual emitted radiance to that of an ideal black body [1].

Emissivity ranges from zero to one, where one would be a black body. The emissivity can also vary with wavelength for any particular substance. As an example, the emissivity for water droplet clouds decreases as the as the wavelength decreases from $10.7 \mu \mathrm{m}$ to $3.9 \mu \mathrm{m}$. When viewing a cloud one can see further into its interior with $3.9 \mu \mathrm{m}$ imagery compared to the imagery of the $10.7 \mu \mathrm{m}$ channel. This phenomenon is shown in Fig. 1. The main reason is that substances that are poor emitters are also poor absorbers for any given wavelength (Kirchoff's Law). Thus a cloud that has low emissivity also has low absorptivity, and any emitted radiation within the cloud has a good chance of escaping.
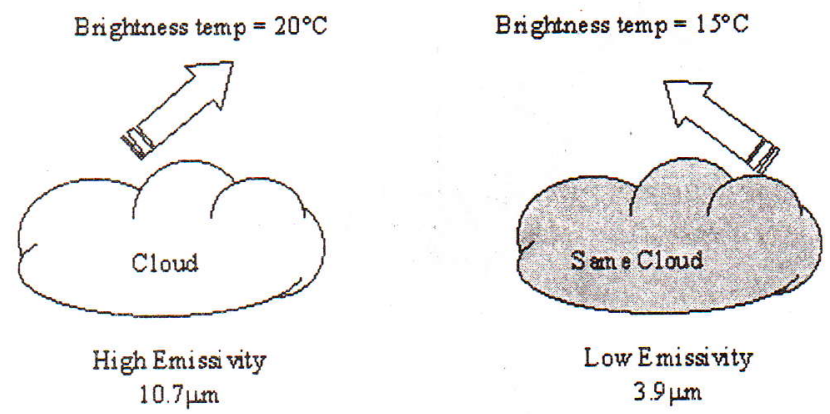

Fig. 1: Example for emissivity of clouds

\section{Numerical calculations}

When we deal with a wave in a medium containing many particles, it is advantageous to consider two extreme cases: tenuous and dense distributions. When the particle density is tenuous, the single scattering approximation can be assumed. As the density increases, first order multiple scattering, multiple scattering and diffusion approximation approaches may be utilized (Fig. 2). In this study the bistatic scattering and emissivity are obtained by averaging over the given orientation distribution. In $[1,3]$ a detailed decription of the theory and the analytical expression of the emissivity (eq) for vegetation is reported [3]. The same method is applicable to clouds, considering that clouds have differently shaped and sized parameters. The emissivity of a cloud is given by the following formula:

$$
e_{q}(\theta)=1-R_{q}(\theta)-T_{q}(\theta)
$$

where

$$
\begin{aligned}
& R_{q}(\theta)=\frac{1}{4 \pi} \times \int_{0}^{\pi} \int_{0}^{2 \pi} \sum_{p=h, v} \frac{\sigma_{p q}^{0}\left(\theta, \theta_{s}, \Phi_{s}-\Phi\right)}{\cos \theta} \sin \theta_{s} \mathrm{~d} \theta_{s} \mathrm{~d}\left(\Phi_{s}-\Phi\right)(2) \\
& T_{q}(\theta)=\frac{1}{4 \pi} \times \int_{0}^{\pi} \int_{0}^{2 \pi} \sum_{p=h, v} \frac{\sigma_{p q}^{\prime 0}\left(\theta, \theta_{s}, \Phi_{s}-\Phi\right)}{\cos \theta} \sin \theta_{s} \mathrm{~d} \theta_{s} \mathrm{~d}\left(\Phi_{s}-\Phi\right)
\end{aligned}
$$




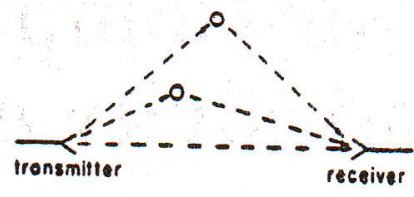

(0)

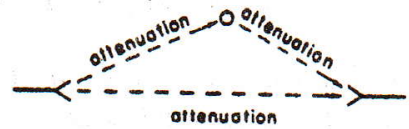

(b)

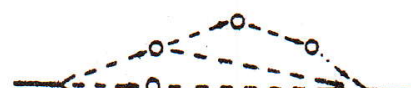<smiles></smiles>

(d)

Fig. 2: (a) Single scattering, (b) first order multiple scattering, (c) multiple scattering and (d) diffusion approximation

where $R_{q}(\theta)$ and $T_{q}(\theta)$ represent the reflected and transmitted portions, respectively, and $\sigma_{p q}^{0}\left(\theta, \theta_{s}, \Phi_{s}-\Phi\right)$ and $\sigma_{p q}^{\prime 0}\left(\theta, \theta_{s}, \Phi_{s}-\Phi\right)$ are computed by means of the matrix-doubling method. The geometry for this scattering is given in Fig. 3.

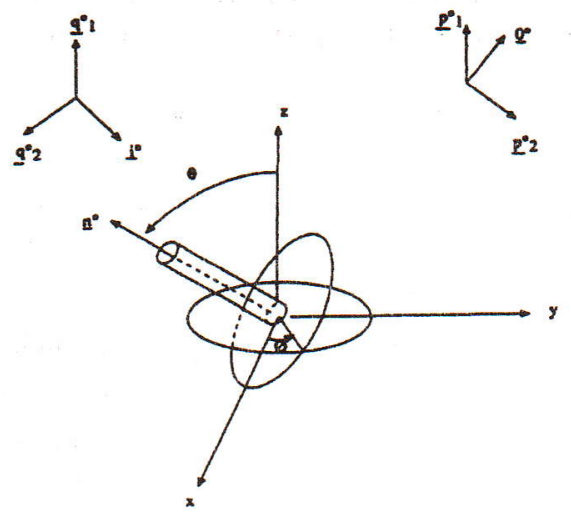

Fig. 3: A rotated lossy dielectric scatterer

Dependencies on $\theta, \theta_{s}$ and $\phi_{s}-\phi$ are discretized in $N_{\theta}$ and $N_{\phi}$ intervals. When cylinders are large with respect to the wavelength, the integral calculations in (2) and (3) can be avoided provided that the absorption cross section is known. In Fig. 4 the average scattering patterns of discs and cylinders with random orientation are shown. They are obtained by averaging the bistatic scattering patterns of an object which assumes different orientations following a uniform distribution in the azimuth (between $0^{\circ}$ and $360^{\circ}$ ) and inclination (between $0^{\circ}$ and $90^{\circ}$ ). The input parameters are listed in Table 1. The direction of the incident wave is always $\theta=45^{\circ}$ and $\phi=0$; at $300 \mathrm{GHz}$, we then take into account cylinders of 135 and $185 \mu \mathrm{m}$ radii and lengths equal to five and seven times the radius, respectively, and circular discs of radius $600 \mu \mathrm{m}$ and thickness $30 \mu \mathrm{m}$. The plots of Fig. 4 represent scattering in the plane of incidence $\left(\phi_{s}-\phi=0\right)$ in the horizontal polarization. The calculated scattering cross sections (which can be obtained by means of surface integrating the bistatic scattering patterns in Fig. 4) are in agreement with these backscatter cross sections which are measured by NATO military radars.

The effects observed in Fig. 4 lead to some computational problems. Fine sampling in the scattering angle is needed if one is to get a correct numerical solution of the integrals in equations (2) and (3). Since the bistatic scattering patterns are mainly forwards, power is either transmitted in the same direction as that of the incidence, or is absorbed. Therefore a scatterer layer may be simply described by a transmission function:

$$
T_{q}^{a}(\theta)=\exp \left(-\frac{n \Delta z \sigma_{a}^{q}(\theta)}{\cos \theta}\right)
$$

Table 1: Input parameters of Figs. 4 and 5

\begin{tabular}{|l|l|l|l|}
\hline Figures & $4(\mathrm{a})$ and 5 & $4(\mathrm{~b})$ and 5 & $4(\mathrm{c})$ and 5 \\
\hline Approximation type & PO & PO & PO \\
\hline Cloud Type & Thin Cirrus & Cirrus & Cirrostratus \\
\hline Scatterer Type & Cylinder & Cylinder & Disc \\
\hline Radius $a(\mu \mathrm{m})$ & 185 & 135 & 600 \\
\hline Cylinder length $2 l(\mu \mathrm{m})$ & 800 & 400 & 30 \\
\hline Disc thickness $T(\mu \mathrm{m})$ & & & 300 \\
\hline Frequency $f(\mathrm{GHz})$ & 300 & 300 & $45^{\circ}$ \\
\hline Incidence angle $(\theta)$ & $45^{\circ}$ & $45^{\circ}$ & $3.13-0.011 \mathrm{j}$ \\
\hline Permittivity $\varepsilon_{\mathrm{r}}$ & $3.13-0.011 \mathrm{j}$ & $3.13-0.011 \mathrm{j}$ & $10^{5}$ \\
\hline Number of scatterers per unit volume $n \Delta v\left(\mathrm{~m}^{-3}\right)$ & $10^{4}$ & $1.2 \times 10^{4}$ & 2000 \\
\hline Cloud-layer height $(\mathrm{m})$ & 500 & 800 & \\
\hline
\end{tabular}




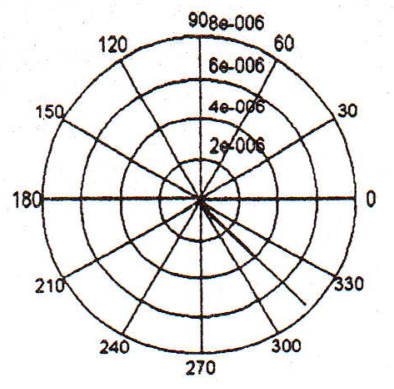

$-\mathrm{a}$ -

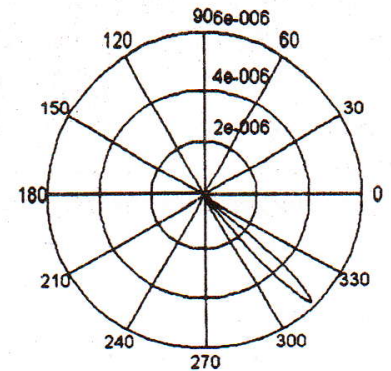

$\cdot b$ -

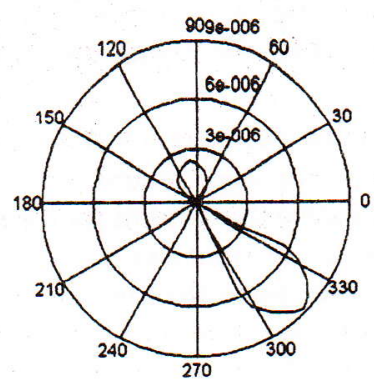

$-c \cdot$

Fig. 4: Average bistatic scattering patterns $\left(\mathrm{cm}^{2}\right)$ of randomly oriented cylinders (a), (b) and discs (c)

assuming $R_{\mathrm{q}}(\theta) \ll \mathrm{T}_{\mathrm{q}}(\theta)$ and $T_{q}(\theta) \cong T_{q}^{A}(\theta)$ leading to the approximation $e_{q}(\theta) \cong 1-T_{q}^{A}(\theta)$. Results are shown in Fig. 5 , where the emissivity at $45^{\circ}$ of a layer filled with equal scatterers with random orientations has been computed as a function of the frequency using the approximate method. Analogous plots are obtained in the vertical polarization. It was seen that the results are in good agreement with the literature [4].

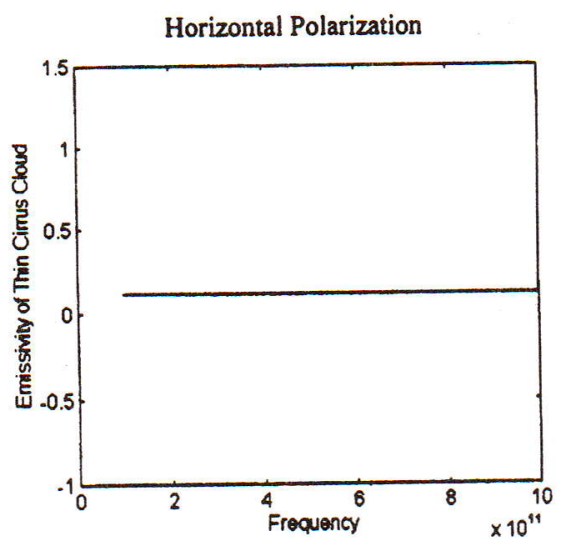

$-a-$

\section{Conclusion}

This work has attemped to give a new formulation of electromagnetic propagation through a cloud. The areas of application of this model cover a wide range from space communication to weather forecasting. It is shown that the bistatic scattering cross section of randomly oriented cylinders becomes more and more peaked in the forwards direction when the cylinder dimensions are large with respect to the wave-

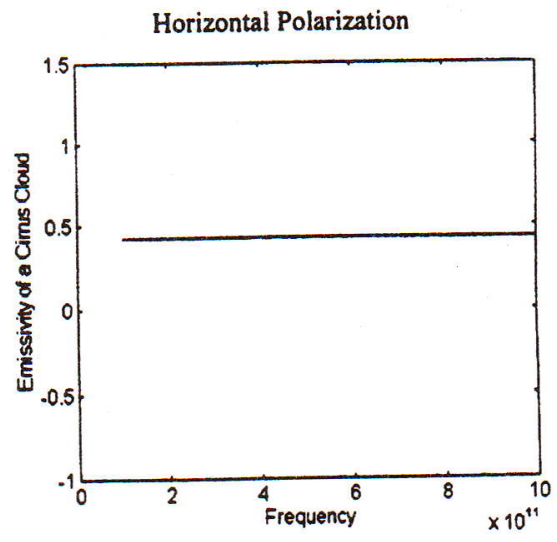

$-b-$

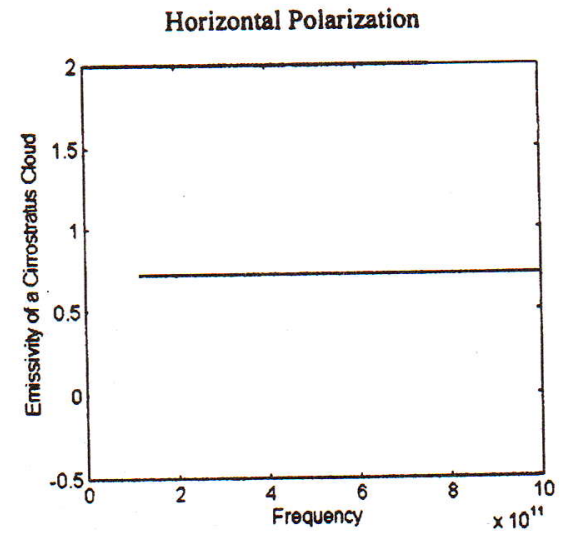

Fig. 5: The emissivity of a layer of cloud as a function of the frequency with horizontal polarization for the parameters given in Table 1 
length. Also in Fig. 4 it is seen that objects tend to behave as absorbers when the object dimensions are close to the wavelength.

It follows that the exact computation of the emissivity of a layer of large cylinders requires very large computational resources. To solve this kind of problem, we have introduced an 'ápproximate' method based on the assumption that cylinders behave like simple absorbers, which makes the use of the cylinder absorption cross section derived in this study. It was seen that the results are in good agreement with the literature [4-6].

\section{References}

[1] Ulaby, F. T. et al: Microwave Remote Sensing. Vol. 1 and 3, New York: Artech House, 1986.

[2] Altshul, E. E. Y: Cloud Attenuation at Milimeter Wavelength. IEEE Trans. On Ant.\&Prop., Vol. 37, No. 199, 1989, p. 1473-1479.

[3] Ferrazzoli, P., Guerriero, L.: Emissivity of Vegetation: Theory and Computational Aspects. In: "Journal of Electromagnetic Waves and Applications", Vol. 10, 1996, p. 609-628.

[4] NASA SUCCESS Mission Overview: http://cloud1.arc.nasa.gov/espo/success

[5] Joe P. Precipitation at the Ground: Radar Techniques: NATO ASI Series, Vol. 145, 1996, p. 227-321.
[6] Painter, A. J. et al: Combined Infrared Emission Spectra and Radar Reflectivity Studies of Cirrus Clouds. IEEE Trans GSRS, Vol. 31, No. 1, 1993, p. 64-69.

Prof. Dr. Selim Seker

phone: +902123581500

$$
\text { +902123581540-1414 }
$$

fax: +902122872465

e-mail: seker@bound.edu.tr

Department of Electrical-Electronics Engineering Bogazici University

34342 Bebek/Istanbul

Turkey

Mustafa Emre Aydemir

Department of Electronics Engineering

Turkish Air Force Academy

Istanbul, Turkey

\section{Gökhan Apaydin}

Department of Electrical-Electronics Engineering Bogazici University

34342 Beybek/Istanbul

Turkey 